\title{
Réseaux traditionalistes catholiques et "réinformation » sur le web : mobilisations contre le « Mariage pour tous » et « pro-vie »
}

\section{Charlotte BLANC}

\section{(2) OpenEdition \\ Journals}

Electronic version

URL: http://journals.openedition.org/ticetsociete/1919

DOI: 10.4000/ticetsociete.1919

Publisher

Association ARTIC

Electronic reference

Charlotte BLANC, « Réseaux traditionalistes catholiques et « réinformation » sur le web : mobilisations

contre le « Mariage pour tous » et « pro-vie » », tic\&société [Online], Vol. 9, Nㅜ 1-2 | 1er semestre 2015 -

2ème semestre 2015, Online since 11 January 2016, connection on 19 April 2019. URL : http://

journals.openedition.org/ticetsociete/1919; DOI : 10.4000/ticetsociete.1919 
tic\&société - 9 (1-2), 2015

Réseaux traditionalistes catholiques et « réinformation » sur le web : mobilisations contre le «Mariage pour tous » et «pro-vie»

Charlotte BLANC

Laboratoire Mica

Université Bordeaux Montaigne

166, cours de la Marne

33000 Bordeaux

charlotteblanc@outlook.fr 


\section{Réseaux traditionalistes catholiques et « réinformation 》 sur le web : mobilisations contre le « Mariage pour tous » et «pro-vie »}

Charlotte BLANC est doctorante en sciences de l'information et de la communication sous la direction du professeur Alain Kiyindou au sein du laboratoire Mica, à l'Université Bordeaux Montaigne. Contractuelle en monitorat, elle assure une charge de cours au département d'information et de communication de l'Université Bordeaux Montaigne.

Résumé : Le présent article traite des réseaux de communication des catholiques traditionalistes sur le web. II se centre en particulier sur les mobilisations «pro-vie » et anti«Mariage pour tous » et sur le traitement de ces thèmes dans la blogosphère. II s'interroge, enfin, sur l'émergence d'un réseau de blogs d'actualité et d'opinion regroupés sous l'expression de « réinfosphère » et sur le rôle et la portée mobilisatrice de cet espace de communication.

Mots-clés: traditionalisme, web, blogosphère, mariage pour tous, pro-vie.

Abstract: Catholic traditionalist networks and re-information on the web: Same-sex marriage and pro-life mobilizations - This article deals with traditional Catholicism networks on the web. It seeks to bring a new perspective into studies of Catholic militantism, especially regarding the theme of family. The article deals with anti-gay marriage and pro-life mobilisations on the Catholic blogosphere. It also questions the rise of a set of ideologically oriented blogs, called "reinfosphere", and their roles in Catholic mobilisation.

Keywords: traditionalist Catholicism, web, blogosphere, samesex marriage, pro-life.

Resumen: Redes tradicionalistas católicas y "re-información" en la web : el "matrimonio para todos" y movilizacion "pro-vida" Este artículo las redes de comunicación católicas tradicionalistas en la web. El artículo comienza con una reflexión sobre el activismo católico en torno cuestiones relacionadas con la familia, para después centrarse en las movilizaciones pro-vida y anti "matrimonio para todos" y en el tratamiento de estos temas en la blogosfera. También se analiza la aparición y el auge de una red de blogs de noticias y de opinión, designada con el término "reinfosfera". Finalmente, se considera el papel y el 
tic\&société - 9 (1-2), 2015

alcance movilizador de semejante espacio de comunicación.

Palabras clave: católicas tradicionalismo, blogosfera, matrimonio gay, movimientos pro-vida.

La notion de traditionalisme catholique renvoie à un ensemble de groupes opposés de façon plus ou moins appuyée à un changement au sein de l'Église catholique, antérieur et postérieur au concile Vatican II $^{1}$ (intransigeantisme, catholicisme intégral, lefebvrisme puis «intégrisme »; Poulat, 1969). Le traditionalisme regroupe un ensemble de mouvances catholiques nées en réaction au tournant de la modernité et centrées sur une conception immuable, mais sélective, de la tradition catholique. Comme le souligne Paul Ricœur dans son introduction à La symbolique du mal (1960) tout ce qui relève de la tradition ne cède pas nécessairement au traditionalisme. Ainsi, la tradition défendue par le traditionalisme catholique coïnciderait avec ce que Gérard Lenclud (1987) qualifie d' « absence de changement dans un contexte de changement ». Certaines tendances traditionalistes sont incluses dans le «giron » de l'Église catholique (comme les communautés rassemblées sous l'égide de la commission Ecclesia Dei ${ }^{2}$, puis Sommorum Pontificum), quand d'autres constituent des groupes dissidents de l'Eglise catholique (les communautés issues de la mouvance lefebvriste ${ }^{3}$ ou les branches sédévacantistes ${ }^{4}$ ).

En outre, la notion de tradition correspond à la « transmission des doctrines religieuses au moyen de différentes sources de la foi chrétienne, Bible, écrits des Pères, des Docteurs de l'Église, des décisions des conciles et des papes » (Suire, 2004) mais exclut de façon plus ou moins importante selon les tendances, l'autorité du concile Vatican II. Elle se manifeste souvent par un attachement à une pratique (le rite tridentin, c'est-à-dire la messe en latin instaurée par le concile de Trente, selon le rite de Saint Pie $V, 1545-1563$ ) et constitue une référence au discours des acteurs sociaux qui s'identifient comme traditionalistes.

Le traditionalisme catholique est une doctrine religieuse qui s'est construite sur un temps long et qui est parvenue à une

${ }^{1} \mathrm{XXI}^{\mathrm{e}}$ concile, deuxième concile œcuménique du Vatican, ouvert en 1962 sous Jean XXIII et achevé en 1965 sous Paul VI.

$2<$ http://www.vatican.va/roman curia/pontifical commissions/ecclsdei/documents/hf i $\mathrm{p}$-ii motu-proprio 02071988 ecclesia-dei en.html>, dernière consultation le $1^{\text {er }}$ août 2015.

${ }^{3}$ Mouvance initiée par Mgr Marcel Lefebvre, évêque fondateur de la Fraternité sacerdotale saint Pie X, fondée en 1970 à Ecône, en Suisse. L'évêque dissident a ordonné en 1988 quatre évêques sans autorisation pontificale.

${ }^{4}$ Terme qualifiant des mouvances traditionalistes qui remettent en cause la question de l'infaillibilité pontificale (décrétée en 1870 par le premier concile du Vatican) et qu considèrent que le siège de Pierre est vacant, sede vacante. Plusieurs tendances existent au sein de cette mouvance sédévacantiste. 
tic\&société - 9 (1-2), 2015

cohérence idéologique fondée sur un argumentaire de la répétition (Rémond, 1989) ; il est un activisme qui se réclame "d'une révélation divine pour organiser l'existence publique » (Milot, 1998, p.159). La notion de tradition, au cœur de la nature même du traditionalisme, est entendue comme volonté de transmettre un message hérité, socialement significatif ${ }^{6}$ (Lenclud, 1987).

Notre démarche de recherche s'inscrit dans un contexte de mobilisations contre le «Mariage pour tous 7 ayant eu lieu de novembre 2012 jusqu'au printemps 2014, et dans celui, plus large, d'une histoire de la militance catholique engagée dans le champ de la famille ${ }^{8}$, de la sexualité et de la bioéthique depuis la loi Neuwirth en $1967^{9}$. Ainsi, comme l'exposent Céline Béraud et Philippe Portier, " parmi les défis qu'a connus le catholicisme français au cours des dernières années, le cas du mariage pour tous constitue [...] un observatoire privilégié des formes de réinvestissement du politique par les catholiques, qui s'accompagnent d'une quête de visibilité dans l'espace public et

${ }^{5}$ En se fondant sur l'hypothèse que « le radicalisme religieux contribue autant à perturber qu'à étendre le champ d'action de la modernité » (p.154) et en remettant en cause les théories de la sécularisation, Micheline Milot cherche à rendre le concept de radicalisme opérationnel et présente les grandes caractéristiques sociologiques communes au traditionalisme, au fondamentalisme et à l'intégrisme, tous les trois entendus comme expression religieuse radicale et activiste.

${ }^{6}$ La notion de tradition est effectivement au centre de l'émergence et de la pérennité du traditionalisme. Ce dernier, entendu comme radicalisme religieux, concept complexe aux réalités culturelles multiples, se construit sur une réinterprétation de la tradition catholique et résulte de la modernisation des sociétés. Ceci place le traditionalisme catholique au centre d'une dialectique tradition - modernité.

${ }^{7}$ Le projet de loi sur l'ouverture des droits du mariage aux couples de même sexe, communément appelé le "Mariage pour tous », a été porté par la ministre Christiane Taubira, Garde des Sceaux. Le projet de loi a été déposé le 7 novembre 2012 et la loi a été promulguée le 18 mai 2013. Les mobilisations contre cette loi, regroupées sous l'expression « Manif pour tous », se sont toutefois poursuivies jusqu'au printemps 2014 dans le but de faire abroger la loi. À cette période (juin 2014) se met en place le plan d'action des $A B C D$ de l'égalité (projet éducatif dont l'objectif est de sensibiliser les enfants à la question de l'égalité entre les femmes et les hommes et de réduire les stéréotypes de genre), contre lequel les opposants au mariage pour tous vont alors se positionner, entraînant une prolongation de la mobilisation ayant pour objet de lutter contre la progression de ce qui est considéré comme une « théorie du genre ».

${ }^{8}$ Nous nous appuyons sur la définition de la famille donnée par le Dictionnaire critique de la sociologie: "Le groupe familial tire son origine du mariage. II comprend le noyau constitué par le mari, la femme et les enfants nés de leur union [...]. Le lien familial est un lien légal, produisant des obligations économiques, religieuses ou autres, notamment en forme de droits et d'interdits sexuels [...] Un des aspects les plus manifestes de l'organisation familiale est l'ensemble des règles qu'il introduit dans la vie sexuelle » (Boudon et Bourricaud, 2006, p.251). Le mariage et la procréation se trouvent au cœur de la structure familiale, d'où l'intérêt de traiter des questions des mobilisations contre le «mariage pour tous » et pro-vie comme inséparables d'une conception de la famille traditionnelle, patrilinéaire. De même, "L'ensemble des transformations qu'a connues la famille depuis le milieu des années 1960 aboutit en fait à une privatisation des rapports familiaux. Considéré autrefois comme une institution fondamentale de la société, le mariage est devenu aujourd'hui un simple contrat d'association » (Etienne et al., 2009, p.199), ce qui est en particulier remis en cause par les mouvances traditionalistes.

${ }^{9}$ La loi du 28 décembre 1967, relative à la régulation des naissances, porte sur la légalisation de la pilule contraceptive. 
médiatique, un observatoire également des transformations internes du catholicisme français en cours depuis plusieurs décennies. II donne ainsi à voir la centralité qu'y ont notamment acquise les enjeux relatifs à la biopolique, devenus pour certains, clercs et laïcs, objets de militance et de mobilisations » (Béraud, Portier, 2015, pp.18-19). Ainsi, la famille, à travers les questions de mariage et de filiation, est une thématique au cœur de l'actualité politique et constitue notre point d'entrée sur les réseaux numériques traditionalistes.

Le caractère revendicatif du traditionalisme catholique, en particulier à la lumière des récents événements sus-nommés, implique d'étudier les acteurs qui le composent comme une minorité active cherchant à avoir une place plus importante dans l'espace public et qui s'engagent dans une «lutte pour la visibilité $"{ }^{10}$ (Voirol, 2005) médiatique. Ainsi le traditionalisme peut être étudié par le prisme des Cultural Studies, en cela qu'il exprime une conflictualité sociale et une tension entre différentes interprétations de la famille.

Le questionnement général de notre recherche concerne les traditionalistes catholiques sur le web, leur place dans l'espace public et leur activisme social et d'opposition visant, notamment par le biais de la «réinformation", à réaffirmer des valeurs issues de «la tradition ${ }^{11}$ ». La réinformation se forme d'un ensemble de blogs découvert lors de la constitution du corpus de sites traditionalistes. Nous tenterons d'apporter un début de définition de ce nouvel objet en dernière partie d'article.

Dans cet article nous nous attelons spécifiquement à répondre aux questions suivantes. Une première démarche consiste à se demander comment aborder la recherche sur la communication religieuse sur le web. Comment étudier les manifestations du traditionalisme sur l'internet? Dans une deuxième démarche, nous nous demandons: quelles sont les caractéristiques et acteurs de la blogosphère traditionaliste ? Enfin, en quoi cette blogosphère est-elle un espace dit de "réinformation » et d'élaboration d'un savoir-faire militant, spécifiquement centré sur les thématiques liées à la famille, cristallisées au moment du Mariage pour tous? Constitue-t-elle un réservoir idéologique ${ }^{12}$ de

\footnotetext{
${ }^{10}$ Le fait que nous travaillons sur des groupes réactionnaires ne doit pas empêcher de les considérer comme s'inscrivant dans une " lutte pour la visibilité » ou une "lutte pour la reconnaissance » (au sens de Honneth, 2000). Ainsi, selon Philippe Gonzalez "On aurait tort de sous-estimer le recours aux "politiques de l'identité » par des courants réactionnaires. II conviendrait dès lors [...] d'entendre la "lutte" comme une catégorie strictement descriptive restituant des dynamiques antagonistes présentes dans l'espace public. Cela implique de ne pas présupposer de la connotation morale de la visibilité en présence » (Gonzalez, 2015, p.257).

${ }^{11}$ La tradition se pose en valeur absolue, étalon-or du système de croyances de ce courant du catholicisme. La charge symbolique de la tradition se trouve a fortiori renforcée par les controverses et mutations actuelles autour des questions de la famille (Mariage pour tous, interruption volontaire de grossesse et moyens contraceptifs, procréation médicalement assistée, gestation pour autrui ou encore études de genre), qui transforment les structures traditionnelles de la famille (essentiellement sur l'alliance et la filiation). 12

L'idéologie est ici entendue dans son acception marxiste (Marx, Engels, 1974) ;
} 
tic\&société - 9 (1-2), 2015

la mobilisation traditionaliste ?

Le Mariage pour tous a fait l'objet d'une mobilisation ecclésiastique importante, ne laissant pas en reste pour autant d'autres acteurs, clercs ou laïcs, comme les traditionalistes. Ainsi, les controverses autour du Mariage pour tous (2012-2013) et les «pro-vie » ont constitué un point d'entrée sur le terrain de recherche. Nous abordons ces questions en excluant la mobilisation sur les réseaux socio-numériques - à la différence de ce qu'ont pu faire Maxime Cervulle et Fred Pailler (2014), à travers l'analyse de la controverse du Mariage pour tous sur Twitter.

Cette recherche s'inscrit dans le champ de la communication religieuse et propose une contribution méthodologique à la recherche de ce champ sur le web.

Sera abordée, dans un premier moment, l'inscription dans le champ de la communication du fait religieux. Nous contextualiserons le questionnement sur les groupes minoritaires et radicaux concernés, pour ensuite réfléchir à la méthode d'observation de la pratique d'une communication religieuse sur le web. Dans un deuxième moment, nous relaterons l'exploration du terrain traditionaliste sur la Toile, des démarches webométriques à un court état des lieux de la présence numérique de ces groupes. Enfin, nous adopterons une focale sur un aspect de la blogosphère traditionaliste, la réinfosphère, afin de saisir les ambitions de ces groupes dans l'espace public, sur le web.

\section{web \\ 1. Aborder la communication religieuse sur le}

\subsection{Enjeux et contexte}

Le traditionalisme catholique s'inscrit dans un contexte de réaffirmation identitaire du fait religieux en Europe observé déjà par Danièle Hervieu-Léger (1999) et François Laplantine (1993) et d'une place de plus en plus importante prise par les religions dans l'espace public (Habermas, 2008) ${ }^{13}$. Cette réaffirmation consiste en une émergence de réactions identitaires issues d'un processus de modernisation des sociétés. Ainsi, la question apparaît marquée à la fois par la dichotomie entre tradition et

Nous posons l'hypothèse de l'existence d'un réservoir idéologique potentiellement actif, et au sens de Louis Althusser (1976), d'une force agissante.

13 Dans l'article "Qu'est-ce qu'une société "post-séculière" ? », Jürgen Habermas (2008) expose l'idée de l'existence de sociétés dites post-séculières qui constitueraient des sociétés « où la religion revendique un rôle public, tandis que recule la certitude séculariste que la religion est vouée à disparaître, à l'échelle mondiale, dans le sillage d'une modernisation accélérée » (p.8), en précisant que «les groupes religieux prennent de plus en plus le rôle de communautés d'interprétation dans la vie politique des sociétés séculières »(p.7). 
modernité, d' autre part par la question identitaire, ce qui la rend singulièrement complexe.

Le traditionalisme cherche à recomposer le lien social (Donegani, 2000 ; Ion, 2000) $^{14}$ et s'est inscrit, à travers les mobilisations Manif pour tous, dans une lutte pour la reconnaissance au sens d'Axel Honneth ${ }^{15}$. Les catholiques traditionalistes, dont les affinités politiques se situent à droite voire à l'extrême droite, sont des acteurs relativement récents dans la sphère publique. Mis à part dans le cadre de la loi sur le Pacs $^{16}$ et des mobilisations pro-vie, ils ont été peu médiatisés depuis l'affaire du schisme lefebvriste ${ }^{17}$. Notons que, pour autant, les mobilisations contre le Mariage pour tous ne naissent pas exnihilo. Ainsi, « faire l'archéologie de cet engagement [...] conduit, selon nous, à s'intéresser à au moins trois mobilisations catholiques antérieures [...] sur des thématiques qui ont fait l'objet d'une intense politisation : l'opposition au Pacs [...] en 1998-1999 ; la "croisade morale" menée d'abord à Rome puis relayée par l'épiscopat français contre une prétendue "théorie du genre" ; l'activisme bioéthique. En ces occasions, des entrepreneurs de morale émergent, des rhétoriques se diffusent et des répertoires d'action s'expérimentent » (Béraud, Portier, 2015, p.22). Le web est un nouveau répertoire d'action du militantisme catholique en général et du traditionalisme en particulier. Cela revient à questionner la réappropriation du dépôt culturel (Lenclud, 1987) qu'est la tradition sur l'internet. Ainsi, comme le présente Gérard Lenclud,

«il ne viendrait à l'idée de personne de considérer comme traditionnel tout ce qui nous vient du passé, la notion de tradition renvoie aussi à l'idée d'un certain domaine de faits, ou, si l'on préfère, d'un dépôt culturel sélectionné. La tradition ne transmettrait pas l'intégralité du passé ; il s'opérerait à travers elle un filtrage ; la tradition serait le produit de ce tri » (Lenclud, 1987, p.112).

Le web est de ce fait un moyen de transmission de la tradition par lequel s'effectue un nouveau tri. Interroger les représentations culturelles de groupes religieux sur le multimédia

\footnotetext{
14 Jean-Marie Donegani (2000) et Jacques lon (2000) s'interrogent tous deux sur la «pluralité des rapports au monde » et «l'engagement dans l'espace public » des religions.

${ }^{15}$ La lutte pour la reconnaissance telle que reprise par Axel Honneth (sur la base d'un travail critique des travaux de Hegel) s'appuie sur l'idée que « l'individu ne réagit pas à l'appropriation des biens par la crainte de se voir un jour menacé dans sa propre existence, mais par le sentiment d'être ignoré par son vis-à-vis. L'attente normative d'une reconnaissance de la part des autres sujets est intégrée dans la structure des relations humaines d'interaction, ne serait-ce que dans la mesure où l'individu s'attend implicitement à être pris en compte de manière positive dans les projets d'autrui » (Honneth, 2000, p.58). La référence à Honneth prend sa pertinence dans l'observation d'un processus d'autonomisation des groupes traditionalistes vis-à-vis de l'Église catholique, et une recherche de visibilité médiatique dans l'espace public.

${ }^{16}$ Pacte civil de solidarité, loi de 1999.

${ }^{17}$ En 1988, Mgr Lefebvre sacra quatre évêques, à Ecône, sans autorisation pontificale, ce qui eut pour effet l'application du Suspens a divinis, ou excommunication par le pape, pour lui et les quatre évêques alors sacrés.
} 
qu'est le web et, par là même, les médiations des identités traditionalistes, dans une logique contemporaine d'expression des particularismes culturels (Milot, 1998; Laplantine, 1993), revient à réévaluer la teneur de la tradition en question (et de ses aspects idéologiques).

\subsection{Questionner le fait religieux sur le web}

La communication religieuse est un champ délicat à traiter tant «il semble que les sciences de l'information et de la communication se soient méfiées -- peut être à juste titre - de la dimension idéologique de l'objet, de la difficulté d'en parler sans $y$ prendre part » (Douyère, Dufour, Riondet, 2014, p.9). L'ambition est, à terme, d'élaborer une conceptualisation de la logique médiatique de la communication religieuse de groupes chrétiens minoritaires et radicaux et de parvenir à une compréhension des processus et dynamiques de communication des acteurs du religieux.

L'étude du phénomène religieux ne saurait être envisagée de façon similaire dans une réalité matérielle et sur le web. Ce qui relève du directement observable dans la première (églises, codes vestimentaires, etc.), est moins évident dans le second cas de figure. Dans l'étude du fait religieux, ainsi que le postule Stéphane Dufour, " penser le sujet au travers du paradigme de fait religieux, et non plus de religion, conduit d'une part à s'intéresser à une réalité objective [...] s'imposant par là même à tous indépendamment des convictions et, d'autre part, à l'arracher aux spéculations théologiques pour le soumettre à l'étude pratique ancrée dans un terrain de recherche. " (Dufour, 2014, pp.96-97). Nous pouvons alors nous demander comment s'intéresser à la réalité objective, aux manifestations du traditionalisme sur l'internet. Dans le cadre d'une recherche sur le web, nous ne pouvons pas nous détacher du socle idéologique du traditionalisme, et ce, afin de pouvoir en identifier les acteurs sur la Toile. À cela s'ajoute le fait qu'une de nos hypothèses s'attache à considérer que la matière idéologique circulant sur le réseau traditionaliste participe d'une mobilisation symbolique ayant un rôle à jouer dans l'action collective. Un des enjeux est alors d'identifier les relations existantes entre acteurs du traditionalisme sur le web. Ainsi, « en visant les formes et les supports de figurativisation ${ }^{18}$ des religions, ce sont des acteurs, des rituels, des discours, des objets, des espaces religieux, etc. que les Sic sont à même de se donner pour objet, plutôt que des religions » (Dufour, 2014, pp.96-97).

II s'agit alors de rendre compte de la structure de communication des traditionalistes catholiques sur le web, d'en saisir les acteurs principaux, les stratégies et intentions de

\footnotetext{
${ }^{18}$ La notion de figurativisation correspond à la permanence d'une figure quel que soit le contexte dans lequel elle se présente.
} 
communication. De ce fait, une analyse relationnelle du web traditionaliste par le truchement d'outils webométriques est préférée. La réalité est ici perçue comme construite sur la base de médiations symboliques entre les acteurs qu'ils produisent dans leurs interactions avec d'autres acteurs. Stéphane Dufour explique que "c'est en analysant les usages du capital symbolique des religions en fonction des structures et du contexte dont elles sont les représentations que les Sic prennent véritablement et utilement le relais des sciences historiques et sociologiques » (Dufour, 2014, p.99). Nous ne cherchons pas à produire une vision exhaustive des formes de présence et des usages des traditionalistes sur le web. Dans une logique interactionniste (qui «se donne pour tâche de reconstruire l'ensemble social à partir des règles simples que l'analyse de petits groupes et l'observation des interactions humaines permettent d'établir », Godin, 2004, p.678) nous avons décidé de suivre les choix opérés par les webmestres dans l'élaboration de leurs blogrolls ${ }^{19}$.

\section{L'exploration du réseau traditionaliste}

\subsection{Méthode de collecte}

Une recherche exploratoire a permis de construire notre objet de recherche. L'observation des réseaux traditionalistes a révélé l'existence d'une blogosphère singulière, la réinfosphère (ou blogosphère de « réinformation »), suivant un terme employé par les acteurs eux-mêmes. Nous nous demandons ici quelles en sont les caractéristiques et en quoi peut-elle constituer un espace dit de « réinformation » et d'élaboration d'un savoir-faire militant. Dans cette recherche nous abordons l'action religieuse sur le web dans une épistémè constructiviste. De même, l'inscription théorique se situe au croisement de la sociologie des mouvements sociaux d'Alain Touraine, des apports postcritiques des cultural studies et de l'anthropologie constructiviste. Nous nous inspirons notamment des travaux d'Eric Macé (2006). Ainsi, "l'objet est l'analyse des mouvements et des contremouvements culturels dans la sphère publique et dans les médiacultures et leurs usages » (Macé, 2006, p.16) L'approche se veut également compréhensive. En ce sens, le web étant un espace de représentation et d'expression (individuelle ou collective), le positionnement ne peut être qu'essentiellement qualitatif et compréhensif. Nous travaillons sur la base d'un matériel idéologique et symbolique (Olivesi, 2009) qui rend délicate une parfaite objectivité en la matière et demande de

${ }^{19}$ Blogroll : liste de liens proposés sur un site web ou un blog dans laquelle sont référencés les sites et blogs conseillés et/ou consultés par le webmestre du blog étudié. 
dépasser une observation de la structure des réseaux (ne s'intéressant qu'aux relations entre les éléments et non aux attributs).

Nous avons employé la méthode d'échantillonnage de réseau ${ }^{20}$ pour laquelle deux indicateurs ont été sélectionnés. Le premier est l'appartenance au catholicisme traditionaliste. Cet indicateur a nécessité l'élaboration d'une grille de lecture sur la base de critères théologiques, liturgiques, idéologiques et ritualistes des traditionalistes catholiques, permettant de vérifier les appartenances idéologiques et confessionnelles. Pour cela un état de l'art a été effectué sur la question et a permis d'identifier des indicateurs du traditionalisme catholique (Perrin, 1989; Rémond, 1989 ; Michel et Sesboüé, 2009 ; Tincq, 2009 ; Senèze, 2008 ; Poulat, 1969 ; Congar, 1976). Cette grille a été nécessaire pour les groupes du réseau n'ayant pas d'appartenance officielle au traditionalisme. Le second critère implique l'opposition au Mariage pour tous et/ou pro-vie ${ }^{21}$. Ce critère a fait l'objet d'une vérification systématique en première lecture des sites et des blogs. L'intention ici était de mettre en avant les particularités des réseaux traditionalistes sur l'internet par le prisme des sites catholiques pro-vie et anti-Mariage pour tous.

La sélection du corpus s'est établie en priorité par le biais de sites internet d'institutions cultuelles (Fraternité sacerdotale Saint Pie X, Fraternité Saint Pierre, Institut Christ-Roi, etc. ; sites employés comme " sites noyaux », c'est-à-dire sites de départ de la recherche) et d'associations mobilisées dans le combat contre le Mariage pour tous et pro-vie (Civitas, SOS Tout-petits). La seconde étape fut de mettre en avant les relations d'un blog à l'autre en passant par les blogrolls. Ce choix des blogrolls est dû principalement au fait que le groupe ou individu en charge du blog se positionne lui-même au sein d'un réseau d'informations, ce qui permet de situer l'internaute dans un champ de références symboliques communes. Le blog de Jeanne Smits ${ }^{22}$ par exemple, présente les liens préférentiels en intitulant la rubrique «Ma géographie du web ». II s'agit donc d'un moyen de se représenter et de se positionner dans un espace symbolique. Le référencement des blogrolls s'est accompagné d'une vérification de l'appartenance de chacun des blogs mentionnés ou listés. Par appartenance nous entendons une filiation spirituelle et d'opinion avec les sites noyaux. Toutefois, la mise en évidence d'un réseau de sites interconnectés nécessite davantage qu'une observation des blogrolls ; c'est pourquoi une collecte des liens hypertextes a

\footnotetext{
${ }^{20}$ Échantillonnage de réseaux (ou boule de neige) : échantillonnage empirique, les sites sont sélectionnés en fonction des liens qu'ils entretiennent avec un site-noyau.

${ }^{21}$ Nous avons fait le choix d'observer la mobilisation en ligne de groupes religieux clairement positionnés contre le mariage pour tous et défenseurs «pro-vie " (c'est à dire contre I'IVG et l'idée d'euthanasie). II ne constitue pas une tentative de généralisation d'un positionnement politique et religieux à l'ensemble des communautés traditionalistes.

22 <http://leblogdejeannesmits.blogspot.fr/>, dernière consultation le $1^{\text {er }}$ septembre 2015.
} 
tic\&société - 9 (1-2), 2015

également été menée, dans une démarche propre aux link studies. Chaque blog mis en évidence par l'observation des blogrolls a été entré dans un crawler $^{23}$ (Jacomy, Ghitalla, 2007), " un robot qui explore et indexe les sites web en laissant le chercheur guider la navigation » (Plantin, 2013). La méthode permet de révéler l'existence de liens hypertextes sur un blog. Le logiciel Gephi ${ }^{24}$ a ensuite permis de visualiser l'ensemble du réseau. Le corpus conservé à ce moment-là comportait 60 sites. La démarche a permis de mettre en évidence certaines caractéristiques morphologiques du réseau. L'outil cartographique a été utile en ce sens au début de notre démarche puisque nous sommes partie d'un terrain (les sites institutionnels) en cherchant à voir vers quels sites ils s'orientaient. En employant les ressources d'hyperliens centrées autour de centres d'intérêts (confessionnels) et visualisables par cartographie, un réseau d'acteurs et des thématiques ont pu être identifiés.

\subsection{Le réseau traditionaliste}

Les blogs révèlent une palette d'acteurs appartenant principalement au domaine associatif, relevant du blog collaboratif et du journalisme amateur. Chaque association s'insère dans un réseau d'associations sous la forme de collectifs. Ainsi, le réseau se subdivise en plusieurs réseaux souvent orientés sur une thématique précise. Le collectif pour l'enfant ${ }^{5}$ regroupe par exemple près de 80 associations nationales et locales. Au sein de ces collectifs, la thématique de la famille a une place privilégiée et constitue un intérêt partagé (Mercklé, 2011) fondé sur un modèle de vie familial traditionnel et en appelle au respect de la « dignité de la personne humaine $\gg 26$.

La blogosphère se divise en diverses tendances; une sphère spirituelle et d'entraide traditionaliste concentre les groupes cultuels. Les traditionalistes des différentes communautés religieuses trouvent sur l'internet des espaces de spiritualité ou de discussion (Forum catholique ${ }^{27}$ ) et d'entraide communautaire (Entraide catholique $\left.{ }^{28}\right)$. Cette sphère contient également des

${ }^{23}$ Crawler: "Outil d'exploration du web qui analyse le contenu et la structure des pages et des liens hypertextes et permet de réaliser des graphes de l'ensemble des sites web sélectionnés par l'utilisateur ou de les classer. " (Barats, 2013, p.124). $<$ http://webatlas.fr/wp/navicrawler/>, >, dernière consultation le $1^{\text {er }}$ septembre 2015.

${ }^{24}<$ http://gephi.github.io/> (dernière consultation le $1^{\text {er }}$ septembre 2015) ; « plateforme interactive de visualisation et d'exploration de graphes et de données complexes », (Barats, 2013, p.210).

$25<$ http://www.collectifpourlenfant.fr/> , dernière consultation le $1^{\text {er }}$ août 2015.

${ }^{26}$ Compendium de la Doctrine sociale de Église, $<$ http://www.vatican.va/roman curia/pontifical councils/justpeace/documents/rc pc jus tpeace doc 20060526 compendio-dott-soc fr.html>, dernière consultation le $1^{\text {er }}$ août 2015.

$27<$ http://www.leforumcatholique.org/>, dernière consultation le $1^{\text {er }}$ août 2015.

$28<$ http://www.entraide-catholique.org/site/index.php $>$, dernière consultation le $1^{\text {er }}$ août 
sites de petites annonces destinées aux fidèles traditionalistes, ainsi qu'un réseau national qui référence tous les réseaux locaux d'entraide sur le territoire français (le réseau Missa ${ }^{29}$ ). Sur les réseaux traditionalistes, une blogosphère politique se distingue ensuite qui se subdivise en plusieurs tendances: des mouvances royalistes (Les Manants du Roi ${ }^{i 0}$ ) et maurrassiennes (Action francaise ${ }^{31}$ ), une tendance identitaire (sous l'impulsion de Novopress ${ }^{32}$ ), et une tendance plus historique et révisionniste.

Une des particularités typologiques du réseau est l'existence d'une blogosphère militante composée d'associations et collectifs structurés autour d'une cause familiale (Pro-vie ou anti-Mariage pour tous). De façon plus large, ces associations s'intéressent à tous les domaines relevant des questions de bioéthique et familiales (hétéroparentalité, interruption volontaire de grossesse). Certains de ces collectifs fonctionnent en autonomie (sans collaboration avec les autres) et, pour des raisons de désaccord sur des questions de fond, ne communiquent pas avec d'autres collectifs ayant pourtant la même cause centrale. Un des principaux points de désaccord au sein des associations se situe au niveau de l'expression de la foi ; certains assument leur identité religieuse quand d'autres la dissimulent afin d'ouvrir la cause aux autres confessions (c'est le cas du Printemps français ${ }^{33}$ ). Dans la lutte contre le Mariage pour tous, le désaccord se fonde sur la question de l'intégration des personnes homosexuelles au combat contre la loi sur le Mariage pour tous. Enfin, le réseau traditionaliste comporte une dernière sphère qui a retenu notre attention de par sa singularité, c'est la sphère auto-dénommée de réinformation, ou autrement appelée réinfosphère.

\section{La « réinfosphère »: un organe de communication \\ 3.1. Présentation de la blogosphère de « réinformation »}

Le réseau de mobilisation traditionaliste n'est donc pas homogène ; malgré cette hétérogénéité, l'ensemble de ce réseau est alimenté par une réinfosphère, blogosphère de « réinformation » des traditionalistes. De l'ensemble des sphères

\footnotetext{
2015.

${ }^{29}<$ http://www.missa.fr/ Le réseau Missa est présenté par le site Entraide Catholique comme un partenaire de la Fraternité sacerdotale saint Pie $X$ et Civitas comme un " site ami ».

$30<$ http://www.lesmanantsduroi.com/; dernière consultation le $1^{\text {er }}$ août 2015.

$31<$ http://www.actionfrancaise.net/craf/> dernière consultation le $1^{\text {er }}$ août 2015.

$32<$ http://fr.novopress.info/>, dernière consultation le $1^{\text {er }}$ août 2015.

$33<$ http://www.printempsfrancais.fr/, >, dernière consultation le $1^{\text {er }}$ août 2015.
} 
déjà évoquées et sur lesquelles nous ne nous étendrons pas ici, émerge en effet une sphère informationnelle à visée réinformationnelle. Blogosphère d'actualité, la réinfosphère se donne, un objectif de relecture de l'actualité à la lumière de la tradition catholique (c'est en particulier le cas de Tradinews ${ }^{34}$ ), plaçant davantage les acteurs qui l'animent dans une logique interprétative de l'actualité (politique, religieuse, culturelle et morale) et donc d'opinion, que dans une réelle démarche d'information. L'intention réinformationnelle exprimée dans la présentation des blogs se fonde sur un postulat de désinformation, jugée systématique, des médias dominants de nature idéologique, voire anti-chrétienne (en attestent l'émergence et la circulation de la notion de « christianophobie » sur la blogosphère).

Les sites obtenus grâce au suivi des liens constituent un pôle de convergence des blogosphères d'extrême droite, d'entraide et militantes qui s'informent sur cette réinfosphère. Certains sites se retrouvent au centre de ce recoupement, comme Le Salon Beige, Novopress, le Blog de Jeanne Smits, Riposte catholique, ou encore Contre-info (site de réinformation du Renouveau Français). En reprenant une partie du travail effectué sur la base des blogrolls, il est possible d'illustrer l'inscription idéologique dans laquelle se positionne tel ou tel blog. Nous prendrons l'exemple ici de TradiNews, dont l'appartenance confessionnelle au traditionalisme est revendiquée. Nous avons procédé à une observation du contenu des blogs. Cet aspect se manifeste par plusieurs critères dont seulement deux seront évoqués ici. Le premier concerne la présentation de soi (en rubrique «Qui sommes-nous ? 》 principalement) et la mise en scène thématique par le biais des rubriques. Voici l'ordonnancement des rubriques du site TradiNews: Chercher, Liens, Cont@ct, Réseaux, CCCSP (Centre Saint-Paul), Le FC (Forum Catholique), Riposte (Riposte Catholique), Le SB (Salon Beige), Métablog, Messes. La mention du Forum catholique, de Riposte catholique et du Salon Beige est représentative de la place que TradiNews donne aux trois plateformes très influentes dans les milieux traditionalistes. Ce sont autant d'indications vers des blogs « amis » de TradiNews à consulter.

Le blogroll de TradiNews ${ }^{35}$ comporte pour l'essentiel des liens vers des blogs institutionnels : Fraternité sacerdotale saint Pierre (FSSP), Fraternité sacerdotale saint Pie X (FSSPX), Institut du Bon Pasteur et d'autres. Ceci le positionne dans une légitimité orthodoxe pour autant « œcuménique » au regard des désaccords existants par exemple entre la FSSP et la FSSPX. Le blog classe ses références en catégories, distinguant les sites et blogs traditionalistes des blogs opposés à la FSSPX ${ }^{36}$. II oriente ainsi l'internaute dans une navigation orthodoxe à travers les sites qualifiés de «tradis » et plus élargie par le bais des sites

$34<$ http://tradinews.blogspot.fr/>, dernière consultation le $1^{\text {er }}$ août 2015.

$35<$ http://tradinews.blogspot.fr/p/links.html > , dernière consultation le $1^{\text {er }}$ août 2015.

${ }^{36} \mathrm{C}$ 'est le cas par exemple de <https://www.stageirites.fr/category/doctrine/>, dernière consultation le 11 septembre 2015. 
«non tradis». TradiNews propose en outre des sources d'information émises par des sites de « résistance » à la FSSPX, ce qui suggère une extension des désaccords entre les différentes mouvances traditionalistes sur le web.

Le fait le plus notable (mais il y en a bien d'autres) est la présence dans ce blogroll de diverses fraternités traditionalistes couvrant toutes les tendances, de la plus modérée à la plus radicale : la Contre-Réforme catholique, la Fraternité sacerdotale saint Pierre, la Fraternité sacerdotale saint Pie X, etc. TradiNews joue alors un rôle de portail informationnel pour l'ensemble de la communauté traditionaliste.

Un autre exemple intéressant que nous pouvons présenter est la catégorisation de liens du blog Le Salon Beige. Ce dernier est présenté comme étant un «blog quotidien d'actualité par des laïcs catholiques ». Ce blog est un cas qui illustre un investissement laïc dans l'information traditionaliste et catholique de façon générale. II est l'exemple d'une désinstitutionnalisation de l'information dans ces milieux catholiques. L'ensemble des blogs du corpus de la réinfosphère est, en effet, géré par des laïcs et de façon non-institutionnelle.

Ce choix d'exemples se justifie en outre par l'intense variété de domaines et de préoccupations que couvre le blog dans son blogroll (Mariage pour tous, pro-vie, christianophobie, FSSPX, blogs d'extrême droite et autres), le positionnant par là-même de façon centrale dans le réseau. Au sein du réseau réinformationnel, Le Salon Beige est mentionné sur l'ensemble des blogs de la réinfosphère (soit directement dans le blogroll soit par le partage de posts), ce qui suggère une forte influence idéologique de ce blog laïc et non institutionnel dans le réseau traditionaliste. De même, le blog propose une typologie des liens qui illustre les intentions de communication. Le blogroll se divise en six rubriques: “Opposition à la loi Taubira " ${ }^{37}$ (comprenant La manif pour tous, les Veilleurs et Objection de conscience), «Blogs et Forums », «Pages d'information », «Analyses et opinion », "Sites de documentation », enfin une rubrique menant à d'autres médias «Audios et vidéos». La première rubrique confirme une opposition au Mariage pour tous. D'autres rubriques sont orientées vers l'accès à de l'information dont les sources émanent de blogs tantôt orientés pro-vie (Le blog de Jeanne Smits $^{38}$, étudiants pro-vie ${ }^{39}$, ou Marche pour la vie, contre le dérèglement bioéthique ${ }^{40}$ ), ou contre la « christianophobie ${ }^{41}$. D'autres sont tournés vers le révisionnisme historique avec Jean Sévilla $^{42}$ par exemple, ou forment un micro-réseau de

\footnotetext{
${ }^{37}$ Nom donné par les traditionalistes à la loi ouvrant les droits du mariage aux personnes de même sexe.

${ }^{38}<$ http://leblogdejeannesmits.blogspot.fr/>, dernière consultation le $1^{\text {er }}$ août 2015.

$39<\mathrm{http}: / /$ etudiantsprovie.com>, dernière consultation le $1^{\mathrm{er}}$ août 2015.

$40<\mathrm{http}: / /$ enmarchepourlavie.fr/> , dernière consultation le $1^{\text {er }}$ août 2015.

$41<$ http://www.christianophobie.fr/>, dernière consultation le $1^{\text {er }}$ août 2015.

$42<$ http://www.jeansevillia.com/?page=accueil>, dernière consultation le $1^{\text {er }}$ août 2015.
} 
tic\&société - 9 (1-2), 2015

réinformation comme Riposte Catholique ${ }^{43}$. Ces blogs fournissent des moyens d'action en vue de mobilisations mais sont essentiellement des sources d'information dont l'objectif est la formation argumentative.

\subsection{La réinfosphère : organe de « réinformation»}

D'une première observation des productions textuelles de ces blogs, et surtout sur la base des présentations qu'ils proposent d'eux-mêmes et de leurs missions, un point essentiel émerge. Leur raison d'être se retrouve dans la conviction d'une domination médiatique et politique incarnée par les grands médias, perçus comme étant de gauche et infiltrés par la francmaçonnerie. Ceux-ci produiraient une information erronée en vue de servir leurs propres intérêts, impliquant la nécessité de produire une «contre » information. La réinformation constitue donc une dynamique de lutte contre la désinformation idéologique volontaire attribuée aux médias dominants; il ne s'agit rien moins, pour les acteurs traditionalistes du web, que de " rétablir la vérité », dans une perspective de "salut » social. Ceci est très important pour les mobilisations pro-vie et antimariage pour tous, car c'est dans cette " réinfosphère » que les associations vont puiser l'information. Elle est centrale, et c'est pourquoi tous les sites qui la composent se retrouvent dans les blogrolls des autres sphères. Les expressions réinfosphère et réinformation sont des appellations internes à la blogosphère traditionaliste. Certains intitulés de blogs témoignent de leur intention: «Contre-Info » (site d'information du Renouveau français), ou «Riposte Catholique : portail de réinformation au quotidien ». Le site Novopress se présente ainsi : "Novopress se donne pour objectif de refaire l'information face à "l'idéologie unique". Mais ce travail de réinformation ne peut pas se faire seul. La complémentarité entre les différentes plateformes doit permettre de développer un véritable écosystème réinformationnel $\gg$.

Une analyse de contenu logico-sémantique de productions textuelles et visuelles de documents de la blogosphère a révélé un intérêt particulier de cette réinfosphère pour les sujets entrant dans la thématique de la famille: le mariage homosexuel, la procréation médicalement assistée (PMA), la gestation pour autrui (GPA), les études de genre, etc. La famille constitue alors un espace symbolique de conflictualité dans lequel se concentrent les crispations identitaires de certains groupes sociaux. L'intérêt porté à la blogosphère se justifie par la forte capacité représentationnelle du dispositif blog qui permet au bloggeur de produire une importante identité déclarative (George, 2009). Les intentions communicationnelles sont alors

\footnotetext{
${ }^{43}<$ http://www.riposte-catholique.fr/>, dernière consultation le $1^{\text {er }}$ août 2015.

44 Les « différentes plateformes » auxquelles Novopress fait allusion sont FdeSouche, Polémia, Nouvelles de France, Info Bordeaux et Minute.
} 
identifiables par le biais d'analyses de contenu des blogs, notamment par le prisme des «présentations de soi » et des rubriques thématiques. II s'agit de saisir les représentations de cette blogosphère de réinformation, cadre d'énonciation de la Tradition catholique et d'interroger sa capacité mobilisatrice.

Des productions textuelles et visuelles ont été traitées par une méthode d'analyse logico-sémantique thématique, notamment sur les sites SOS Tout-Petits, Le Blog de Jeanne Smits, France Jeunesse Civitas ou encore Le salon Beige et Riposte catholique. La binarité de la rhétorique traditionaliste sur les sujets du Mariage pour tous et de l'IVG pourrait sans doute être appliquée au reste des thématiques dominantes de la réinfosphère (telles que la christianophobie, le combat de la tradition, l'anti-féminisme, le genre, le complot judéomaçonnique), mais une analyse de discours à la fois plus élargie et plus approfondie serait nécessaire. Nous nous sommes contentée des deux thèmes indiqués ci-dessus.

Ainsi, outre une dualité opposant les tenants du mal aux tenants du bien, il est notable que le discours traditionaliste est un discours du contre, qui se construit en opposition, en réaction à un événement extérieur, élément déjà mis en avant par René Rémond (1989). Béatrice Fracchiolla (2015, p.22) confirme dans un article nos conclusions préliminaires. Elle met en effet en avant la nature oppositionnelle du discours contre le Mariage pour tous et parle de l' " émergence d'un contre-discours d'action collective ».

Les techniques d'argumentation des acteurs de la réinfosphère peuvent être classées en plusieurs catégories de procédés. Se dégage tout d'abord une argumentation complotiste (le reste du monde contre nous), une apologie du combat ensuite (nous contre le reste du monde), qui trouve racine dans une logique d'oppression et donne naissance à une rhétorique martyrologique. Celle-ci est illustrée à force d'images (fœtus morts pour les pro-vie, manifestants gazés pour les opposants au Mariage pour tous) faisant des militants les victimes d'un régime répressif. Ce dernier point justifie, l'existence même de la blogosphère de réinformation qui joue ici un rôle de tribune publique.

L'attitude martyrologique, assez semblable aux procédés rhétoriques employés par Mgr Lefebvre dans les années 1990, s'illustre par une glorification de la France catholique (exaltation du passé) justifiant la rhétorique religieuse du châtiment divin. Les auteurs des posts proposent également, dans une hybridation de référents de l'écologie et du développement durable, une théologie anthropologique soutenue par des apports de la science sur la biologie naturelle (on justifie l'état actuel de la famille : «papa + maman = enfant » par la réalité biologique des sexes et sur la base de la politique de l'Église catholique sur cette question, mais aussi en invoquant l'avenir écologique de l'espèce humaine). Notons un élargissement du thème du Mariage pour tous aux questions de la gestation pour autrui et de 
la « théorie » du genre, fortement réfutée (le genre n'est pas une construction sociale mais une réalité biologique) qui pourront, ultérieurement, être analysés.

Plusieurs points fondamentaux semblent se dessiner pour la recherche que nous poursuivons, et il nous est possible ici de tracer quelques pistes. La dimension ré-informationnelle des blogs traditionalistes, fortement connectés avec la blogosphère d'extrême droite, manifeste la dimension contre-hégémonique de I'intention communicationnelle des acteurs de la réinformation. Ici se justifie l'emploi dans cette recherche de références aux travaux menés dans le champ des cultural studies, bien qu'elles puissent apparaître quelque peu paradoxales en ce contexte.

Les traditionalistes catholiques en tant que groupe social (bien que non homogène) sont porteurs de revendications sociales et manifestent leur envie de réhabiliter - sinon de restaurer l'espace public, le discours religieux. Se considérant presque comme des groupes subalternes (au sens de Nancy Fraser, 2012), relégués (par la laïcité notamment) à une nonmédiatisation, ils sont en recherche de légitimité et visent à contre-dire le système médiatique contemporain dans la perspective d'une affirmation de "la vérité » issue de la tradition.

Une similarité avec des groupes sociaux étudiés par les cultural studies est intéressante à observer. Peut-être alors que dans une logique médiaculturelle ${ }^{45}$ nous pourrions envisager d'opter pour une double lecture entre un « médiactivisme » dans lequel nous pouvons appréhender les logiques médiatiques contre-hégémoniques ou expressivistes (Cardon et Granjon, 2013 ; Allard et Blondeau, 2007) des traditionalistes catholiques et une lecture de la recomposition des pratiques médiatiques, relationnelles et activistes de ces groupes dans un contexte de changement social propre aux cultural studies (qui entendent rendre compte " de l'effet des reconfigurations des rapports sociaux sur la culture autant que des manifestations culturelles du changement », Cervulle, Quemener, 2015, p.7). II est ensuite notable que ces mêmes acteurs produisent un discours de l'action collective à visée performative. Les traditionalistes catholiques, à l'image de ce que Béatrice Fracchiolla (2015) soulève à propos de la "Manif pour tous », souhaitent réinvestir la sphère publique et notamment en « resignifiant la filiation ».

\section{Conclusion}

Cet article entend tirer les premières conclusions d'une observation de l'action collective et du militantisme traditionaliste catholique sur l'internet, par le prisme de la blogosphère de réinformation. Le catholicisme traditionaliste est pluriel et

\footnotetext{
${ }^{45}$ Les « médiacultures », concept développé par Eric Macé et Eric Maigret, renvoient à l'« ensemble des rapports sociaux et des expériences médiatisées par les représentations médiatiques et leurs usages » (Macé, 2006, p.12).
} 
regroupe des tendances diverses mais sur l'internet il en résulte que tous ses mouvements convergent vers la défense d'un idéal traditionnel de la famille. Le Mariage pour tous réactive des frustrations déjà présentes, mais estompées depuis lors, pour les lois sur l'IVG et le Pacs, chez des minorités de plus en plus actives et décomplexées par l'affirmation de la foi religieuse dans l'espace public.

Dans ce processus de médiatisation de l'action, les traditionalistes prolongent sur le web leur héritage contestataire. L'internet représente pour ces minorités religieuses le moyen de renforcer un lien communautaire, de promouvoir un positionnement idéologique minoritaire ainsi que de conserver une visibilité, et, par là, un lien avec le reste de la société, ou tout du moins des débats qui l'animent. Dans notre volonté d'observer les lieux de la mobilisation informationnelle des traditionalistes catholiques et dans une hétérogénéité d'acteurs et d'intentions communicationnelles (associatifs, militants, institutionnels - fraternités -, etc.), nous avons pu voir émerger une blogosphère aux caractéristiques bien particulières.

La blogosphère dite de réinformation est constituée à ce jour (dans notre corpus) d'une vingtaine de blogs d'actualité et d'opinion. L'objectif affiché est de fournir une information alternative au journalisme des médias de masse, dans une démarche de réaffirmation identitaire. De la même façon le militantisme traditionaliste se détache des stratégies de communication institutionnelles de l'Église catholique et fait de la réinfosphère un nouvel "écosystème" catholique principalement organisé par des laïcs, aux moyens d'action variés (pétitions en ligne, organisation d'événements entre organisations laïques, apprentissage de techniques de communication, vente de goodies - produits dérivés - pour les manifestations, etc.).

Ainsi, cet ensemble de blogs constitue un terrain foisonnant de possibilités d'études, tant dans les stratégies de communication qu'en matière d'analyse de l'intention communicationnelle et de répertoires d'actions. Les acteurs du traditionalisme (clercs comme laïcs) expriment sur cette blogosphère une volonté de participer au débat public. Le mouvement de politisation récente du collectif «Manif Pour tous » procède d'une même logique.

\section{Références bibliographiques}

ALLARD L. et O. BLONDEAU, 2007, « L'activisme contemporain : défection, expressivisme, expérimentation », Rue Descartes, Paris, Collège international de philosophie, vol.1, n 55, pp.47-58.

ALTHUSSER L., 1976, « Idéologie et appareils idéologiques 
d'état (notes pour une recherche) », La Pensée, n¹51, dans Louis Althusser, Positions (1964-1975), Paris, Les éditions Sociales, pp.67-125.

BARATS C. (dir), 2013, Manuel d'analyse du web, Paris, Armand Colin.

BERAUD C. et P. PORTIER (dir), 2015, Métamorphoses catholiques, Acteurs, enjeux et mobilisations depuis le mariage pour tous, Paris, éditions de la Maison des sciences de l'homme.

BOUDON R. et F. BOURRICAUD, 2006, Dictionnaire critique de la sociologie, Paris, PUF.

CARDON D. et F. GRANJON, 2013, Médiactivistes, Paris, Presses de Science Po ( $2^{\mathrm{e}}$ édition augmentée).

CERVULLE M. et N. QUEMENER, 2015, Cultural Studies, Théories et Méthodes, Paris , Armand Colin.

CERVULLE M. et F. PAILLER, 2014, «\#mariagepourtous: twitter et la politique affective des hashtag ", Revue française des sciences de l'information et de la communication, $\mathrm{n}^{\circ} 4$.

CONGAR Y., 1976, La crise dans l'église et Mgr Lefebvre, Paris, Le Cerf.

DONEGANI J.-M., 2000, «Identités religieuses et pluralités de rapports au monde », dans P. BRECHON, DURIEZ B. et ION J. (dir), Religion et action dans l'espace public, Logiques politiques, L'Harmattan, pp.211-224.

DOUYERE D., S. DUFOUR et O. RIONDET (dir), 2014, MEl, n³8, « Religion et communication », Paris, L'Harmattan.

DUFOUR S., 2014, « La question du religieux comme espace d'énonciation ", dans D. DOUYERE, S. DUFOUR et O. RIONDET (dir), 2014, MEl, n³8, «Religion et communication », Paris, L'Harmattan.

ETIENNE J., F. BLOESS, J.-P. NORECK et J.-P. ROUX, 2009 Dictionnaire de sociologie, Paris, Hatier.

FRACCHIOLLA B., 2015, «Violence verbale dans le discours des mouvements antagonistes : le cas de "Mariage pour tous" et de "Manif pour tous" ", Argumentation et analyse de discours, La revue électronique du groupe Adarr, $\mathrm{n}^{\circ} 14$, <http://aad.revues.org/1940>, dernière consultation le 25 avril 2015.

FRASER N., 2012, «La lutte pour l'interprétation des besoins », dans Le féminisme en mouvements, des années 1960 à l'ère néolibérale, trad. par E. Ferrarese, Paris, La Découverte.

GEORGE F., 2009, «Représentation de soi et identité numérique. Une approche sémiotique et quantitative de l'emprise culturelle du web 2.0 », Réseaux, vol.2, n¹54, pp.165-193.

GODIN C., 2004, Dictionnaire de philosophie, Poitiers, éditions du Temps, Fayard. 
GONZALEZ P., 2015, "Montrer les minarets pour imposer une Suisse "chrétienne". Les sources évangéliques d'une initiative populaire », dans J. STAVO-DEBAUGE, P. GONZALEZ et R. FREGA (dir), Quel âge post-séculier? Religions, démocraties, sciences, Paris, éditions EHESS, «Raisons pratiques ».

HABERMAS J., 2008, «Qu'est-ce qu'une société "postséculière" ? », Le Débat, n¹52, pp.4-15.

HONNETH A., 2000, La lutte pour la reconnaissance, trad. P. Rusch, Paris, Le Cerf.

ION J., 2000, «L'évolution des modes d'engagement dans l'espace public », dans P. BRECHON, B. DURIEZ et J. ION (dir), Religion et action dans l'espace public, Paris, L'Harmattan, pp.199-210.

JACOMY M. et F. GHITALLA, 2007, «Méthodologies d'analyse de corpus en Sciences humaines à l'aide du Navicrawler ", dans D. DIMINESCU (dir), Rapport final Programme TIC et Migrations, Paris, Fondation de la Maison des Sciences de l'Homme, $<$ http://webatlas.fr/wp/share/navicrawler/Guide\%20m\%E9thodo\% 20NC\%202007.pdf >, dernière consultation le 7 avril 2015.

LAPLANTINE F., 1993, «Recomposition du religieux et réaffirmation identitaire dans l'Europe de la fin du $X X^{e}$ siècle », Civilisations, revue internationale d'anthropologie et de sciences humaines, vol.42, $\mathrm{n}^{\circ 2}$, pp.77-89. $<$ http://civilisations.revues.org/2302>, dernière consultation le 7 avril 2015.

LENCLUD G., 1987, « La tradition n'est plus ce qu'elle était... Sur les notions de tradition et de société traditionnelle en ethnologie », Terrain, n`9, pp.110-123.

MACÉ É., 2006, Les imaginaires médiatiques, une sociologie postcritique des médias, Paris, éditions Amsterdam.

MARX K. et F. ENGELS, 1974, L'idéologie allemande, trad. R. Cartelle et G. Badia, Paris, éditions Sociales.

MERCKLE P., 2011, Sociologie des réseaux sociaux, Paris, La Découverte.

MICHEL F. et B. SESBOÜE, 2009, De Mgr Lefebvre à Mgr Williamson, Anatomie d'un schisme, Paris, Lethielleux, DDB.

MILOT M., 1998, «Religion et intégrisme, ou les paradoxes du désenchantement du monde ", Cahiers de recherche sociologique, $\mathrm{n}^{\circ 30}$, pp.153-178.

OLIVESI S., 2004, Questions de méthode, Paris, L'Harmattan.

PERRIN L., 1989, L'affaire Lefebvre, Paris/Montréal, Le Cerf / Fides.

PLANTIN J.-C., 2013, « D'une carte à l'autre: le potentiel heuristique de la comparaison entre graphe du web et carte 
géographique », dans C. BARATS (dir), Manuel d'analyse du web, Paris, Armand Colin, pp. 228-243.

PORTIER P., 2002, « Le mouvement catholique français au $X X^{e}$ siècle. Retour sur un processus de dérégulation », dans J. BAUDOUIN et P. PORTIER (dir), Le mouvement catholique français à l'épreuve de la pluralité, Enquête autour d'une militance éclatée, Rennes, Presses universitaires de Rennes.

PORTIER P., 2008, "L'engagement des catholiques en France au $X X^{e}$ siècle. Transformation d'un régime de présence », La foi d'un peuple.

POULAT É., 1969, Intégrisme et catholicisme intégral, un réseau secret international antimoderniste: La "Sapinière », 1909-1921, Paris, Casterman.

RÉMOND R.,1989, «L'intégrisme catholique. Portrait intellectuel », Études, vol.370, n¹, pp.95-105.

RICOEUR P., 1960, Philosophie de la volonté II, Finitude et culpabilité 2, La symbolique du mal, Paris, Aubier.

SENEZE N., 2008, La crise intégriste, Vingt ans après le schisme de Mgr Lefebvre, Paris, Bayard.

SUIRE E., 2004, Vocabulaire historique du christianisme, Armand Colin.

TINCQ H., 2009, Catholicisme, Le retour des intégristes, Paris, CNRS éditions.

VOIROL O., 2005, «Les luttes pour la visibilité », Réseaux, n॰129-130, pp.89-121. 